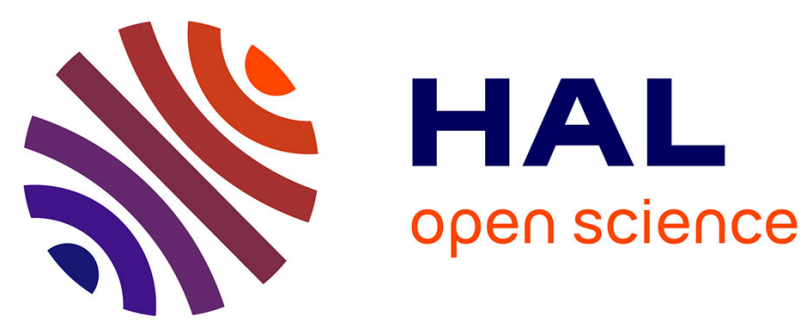

\title{
Application of classical models of chirality to surface second harmonic generation
}

\author{
François Hache, H. Mesnil, Marie-Claire Schanne-Klein
}

\section{To cite this version:}

François Hache, H. Mesnil, Marie-Claire Schanne-Klein. Application of classical models of chirality to surface second harmonic generation. Journal of Chemical Physics, 2001, 115 (14), pp.6707. 10.1063/1.1404983 . hal-00837033

\section{HAL Id: hal-00837033}

https://hal-polytechnique.archives-ouvertes.fr/hal-00837033

Submitted on 19 May 2014

HAL is a multi-disciplinary open access archive for the deposit and dissemination of scientific research documents, whether they are published or not. The documents may come from teaching and research institutions in France or abroad, or from public or private research centers.
L'archive ouverte pluridisciplinaire HAL, est destinée au dépôt et à la diffusion de documents scientifiques de niveau recherche, publiés ou non, émanant des établissements d'enseignement et de recherche français ou étrangers, des laboratoires publics ou privés. 


\title{
Application of classical models of chirality to surface second harmonic generation
}

\author{
F. Hache, ${ }^{\text {a) }}$ H. Mesnil, and M. C. Schanne-Klein \\ Laboratoire d'Optique et Biosciences-CNRS/INSERM/Ecole Polytechnique/ENSTA, 91128 Palaiseau Cedex, \\ France
}

(Received 12 September 2000; accepted 30 July 2001)

\begin{abstract}
Two classical models (Kuhn and Kauzmann) are extended to calculate the second-order nonlinear response of an isotropic layer of chiral molecules. Calculation of the various nonlinear susceptibilities (electric dipolar, magnetic dipolar, and electric quadrupolar) is performed and applied to the derivation of the second harmonic field radiated by the molecules. It is shown that the two models give strikingly different results about the origin of the chiral response in such experiments. Previously published results are analyzed in view of this calculation which allows to understand the different interpretations proposed. This calculation emphasizes the interest of surface second harmonic generation to access information about the microscopic origin of optical activity in chiral molecules. () 2001 American Institute of Physics. [DOI: 10.1063/1.1404983]
\end{abstract}

\section{INTRODUCTION}

Chiral molecules which have no plane of symmetry and occur as right- or left-handed enantiomers are very intriguing from a physicist's viewpoint. They display chiroptical properties such as optical rotatory dispersion or circular dichroism that are currently used to control chirality of molecules. The microscopic origin of this optical activity lies in the nonlocality of the light-matter interaction; one must consider the variation of the electric field associated to the light across the spatial extension of the molecule. By such nonlocal effects, the light is able to probe the spatial organization of the molecule and is sensitive to the handedness of the molecular arrangement. As far as linear optics in isotropic liquids is concerned, nonlocality translates into the influence of the magnetic dipole on the light-matter interaction and molecules must possess together an electric and a magnetic allowed dipolar transition to display optical activity.

Surface second harmonic generation (SHG) has been used for some times to study thin layers of chiral molecules and has proved to be very sensitive to the chirality and to bring new interesting features. ${ }^{1-5}$ In particular, one can get a chiral signature with purely electric dipolar response without considering nonlocal effects. This feature is due to the fact that second-order optics involves three electromagnetic fields that can probe the three directions of space. Such phenomenon has been utilized to explain experimental results such as the rotation of the polarization of the second harmonic beam with respect to the fundamental one on a enantiomerically-pure dihydroxy-binaphtyl surface. ${ }^{2}$ This electric dipolar response has, however, proved not to be sufficient to explain all the results obtained in SHG experiments on chiral surfaces. In particular, careful analyses of the experimental results, especially in terms of the phase of the nonlinear susceptibilities, have shown that pure electric ef-

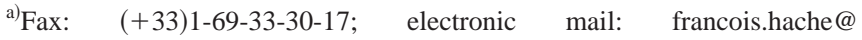
polytechnique.fr
}

fects cannot account for all experimental cases. ${ }^{4,5}$ Indeed, nonlocal effects exist and one must introduce electric and magnetic second-order responses which are both comprised of chiral and achiral components to interpret such experiments. ${ }^{3,5}$ Chiral components are those which cancel out if there exists a plane of symmetry on the surface whereas achiral ones are those which do not cancel. One of the striking features of SHG on chiral surfaces is the following: Playing around with the polarizations of the fundamental or the harmonic beams, it is possible to separate the electric and magnetic contributions on one hand and the achiral and chiral ones on the other hand. Utilizing this procedure, Kauranen et al. showed for example that magnetic contributions were dominant in chiral polymers whereas electric ones were sufficient for helicenebisquinone. ${ }^{6}$

The difference between these experimental results is to be sought in the microscopic origin of the optical activity. Two main classical approaches have been proposed to model the mechanisms of optical activity: ${ }^{7}$ In the coupled-oscillator model, the optical activity arises from the coupling of two separate, noncollinear oscillators. ${ }^{8}$ In the one-electron model, an electron is bound on a helix giving electric and magnetic characters to the optical transitions. ${ }^{9}$ The former model has been successfully applied to describe excitonic coupling and the latter helical polymers for example. However, extension of these models to second-order nonlinear optics has not been fully undertaken. It is the purpose of the present paper to introduce nonlinearity in these two models so as to gain insight in the origin of the nonlinear optical activity and to understand better the differences observed in the previously reported experimental results. In the next section, we will first introduce the general framework; starting from the classical equations of motion and taking nonlocal effects into account, we explain how to get the microscopic hyperpolarizabilities and the macroscopic nonlinear susceptibilities and to calculate the second harmonic radiation. This calculation is then applied to the Kuhn (coupled-oscillators) model (Sec. 
III) and to the Kauzmann (helix) one (Sec. IV). These two calculations give very different results as for the origin of the second-order optical activity. Finally, Sec. V provides a discussion in relation with former experimental results.

\section{GENERAL FRAMEWORK}

In this section, we want to give a general account of the calculation of the second harmonic generation by an isotropic layer of chiral molecules, starting from the microscopic response of individual molecules. SI units are used throughout this calculation. To begin with, one considers the classical equations of motion for the electrons in the system under consideration. The internal forces which constrain this motion depend on the particular model, as described in the following sections. On the other hand, the driving force always originates from the Lorentz force acting on the electrons. Note that only the electric field plays a role as the magnetic term is perpendicular to the electron motion. The following treatment is therefore quite general and applicable to many cases.

First of all, as we are interested in the chiroptical phenomena, we must introduce nonlocal effects in the calculation. This is readily done by expanding the electric field along the spatial directions,

$$
\mathbf{E}=\mathbf{E}(0)+\mathbf{r} \cdot \nabla \mathbf{E} \text {. }
$$

Use of this first-order expansion is justified as the molecular extension is small compared to the wavelength of the light. Higher-order terms are therefore negligible.

So as to introduce nonlinear response in the system, we suppose that the internal forces can be described by anharmonic oscillators. In that case, considering a monochromatic electric field oscillating at fundamental frequency $\omega$, the electronic response displays terms which oscillate at the second harmonic frequency $2 \omega$. From this second harmonic motion, it is possible to calculate the induced electric dipole and, according to Eq. (2.1), we write

$$
\mu_{i}(2 \omega)=\epsilon_{0} \beta_{i j k}^{e e e} E_{j}(\omega) E_{k}(\omega)+2 \epsilon_{0} \widetilde{\beta}_{i j k l}^{e e q} E_{j}(\omega) \nabla_{k} E_{l}(\omega) .
$$

Beside the usual electric dipolar hyperpolarizability $\beta^{e e e}$, we have introduced a nonlocal one $\widetilde{\beta}^{\text {eeq }}$ that takes the electric field gradient contribution into account. We neglect the terms with two gradients as they correspond to higherorder contributions. Note that there is no particular symmetry for the rank-4 tensor $\widetilde{\beta}^{e e q}$ and that, for example, $\widetilde{\beta}_{i j k l}^{e e q}$ $\neq \tilde{\beta}_{i j l k}^{e e q}$ in general. However, we can rewrite Eq. (2.2) by symmetrizing and antisymmetrizing the gradient term,

$$
\begin{aligned}
\mu_{i}(2 \omega)= & \epsilon_{0} \beta_{i j k}^{e e e} E_{j}(\omega) E_{k}(\omega)+2 \epsilon_{0} \beta_{i j k}^{e e m} E_{j}(\omega) B_{k}(\omega) \\
& +2 \epsilon_{0} \beta_{i j k l}^{e e q} E_{j}(\omega) \nabla_{k} E_{l}(\omega) .
\end{aligned}
$$

This expression corresponds to the usual multipolar expansion where beside the electric dipole term, one has the magnetic dipole and the electric quadrupole ones. The factors 2 take into account the fact that the magnetic field (or the gradient term) can originate from one of the two fundamental photons. The tensor $\beta^{e e q}$ is now symmetrical with respect to the inversion of the last two indices. We choose to

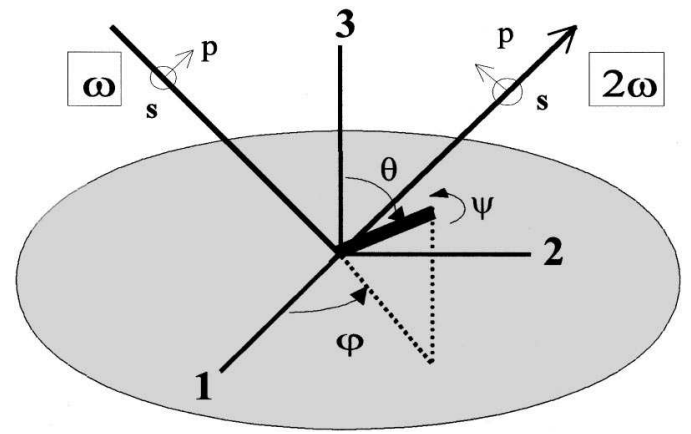

FIG. 1. Geometry of surface SHG. The thick bar represents a molecule referenced by the Euler angles.

first evaluate the tensor $\tilde{\beta}^{e e q}$ and then deduce $\beta^{e e m}$ and $\beta^{e e q}$, as the origin of the nonlocality appears more readily.

Beside this induced electric dipole, we must also calculate the induced magnetic dipole and electric quadrupole,

$$
\begin{aligned}
& m_{i}(2 \omega)=\epsilon_{0} \beta_{i j k}^{m e e} E_{j}(\omega) E_{k}(\omega), \\
& Q_{i j}(2 \omega)=\epsilon_{0} \beta_{i j k l}^{q e e} E_{k}(\omega) E_{l}(\omega) .
\end{aligned}
$$

The above expressions describe the second-order response of an individual molecule. To get the total response of an assembly of such molecules, we have now to average these expressions over the distribution of molecules and to derive the macroscopic electric and magnetic polarizations as well as the quadrupolarization. Nonlinear susceptibilities are then defined by connecting these latter to the electric and magnetic fields.

Let us consider a surface perpendicular to the axis 3 in the laboratory frame (Fig. 1). Considering the usual Euler angles $\theta$ (polar angle), $\varphi$ (azimuthal angle), and $\psi$ (rotation angle) to describe the molecular orientation, we suppose that the last two angles are uniformly distributed whereas $\theta$ is fixed. This corresponds to a simple isotropic surface. If the molecules in the layer are not chiral, the symmetry is $C_{\infty v}$, all the planes perpendicular to the surface become symmetry planes. For chiral molecules, such symmetries are no longer allowed and the symmetry is $C_{\infty}$. Calculation of the macroscopic nonlinear susceptibilities follows the general rules of tensor analysis and one has, for example,

$$
\chi_{I J K}^{e e e}=N\langle(\mathbf{I} \cdot \mathbf{i})(\mathbf{J} \cdot \mathbf{j})(\mathbf{K} \cdot \mathbf{k})\rangle_{\mathrm{av}} \beta_{i j k}^{e e e},
$$

where $i, j, k(=x, y, z)$ refer to the molecular frame and $I, J, K(=1,2,3)$ to the laboratory one, \langle\rangle$_{\text {av }}$ representing the averaging operation. $N$ is the number of molecules per unit surface. Similar relations exist to connect the nonlinear susceptibilities $\chi^{e e m}, \chi^{m e e}, \chi^{e e q}$, and $\chi^{q e e}$ (the latter two being rank-4 tensors) to their microscopic counterparts. It is possible to reduce the number of nonvanishing independent components by taking the symmetry of the surface into account. ${ }^{10}$ The results in the case of SHG are given in Table I. Note that for $\chi^{e e e}, \chi^{\text {mee }}$, and $\chi^{q e e}$, the last two indices can be interchanged without changing the value of the component as both indices refer to the same (fundamental) frequency, further reducing the number of nonzero components. It is not true for the first two indices of $\chi^{e e m}$ and $\chi^{e e q}$ which refer to the harmonic and fundamental frequencies, respec- 
TABLE I. Nonvanishing components of the nonlinear susceptibilities for $C_{\infty v}$ and $C_{\infty}$ symmetries.

\begin{tabular}{lll}
\hline \hline & \multicolumn{1}{c}{ Achiral components $C_{\infty v}$} & \multicolumn{1}{c}{ Chiral components $C_{\infty}$} \\
\hline$\chi^{\text {eee }}$ & $333,311=322,113=131=223=232$ & $123=132=-213=-231$ \\
$\chi^{\text {eem }}$ & $123=-213,132=-231,312=-321$ & $333,311=322,113=223,131=232$ \\
$\chi^{\text {mee }}$ & $123=132=-312=-231$ & $333,311=322,113=131=223=232$ \\
$\chi^{\text {eeq a }}$ & $1111,3333,1122,1212=1221,1133$, & $1222,2122,2212=2221,1233$, \\
& $1313=1331,3311,3131=3113$ & $1323=1332,3123=3132$ \\
$\chi^{\text {qee a }}$ & $1111,3333,1122,1212=1221,1133$, & $1222=2122,2212,2221,1323=3123$, \\
& $1313=3113,1331=3131,3311$ & $1332=3132$ \\
\hline
\end{tabular}

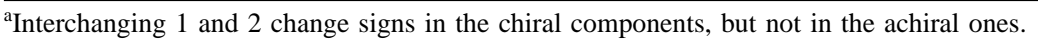

tively. In the first column, the "achiral" components are given, i.e., the components that do not vanish for a $C_{\infty v}$ symmetry. In the second column, we have written the additional components that do not vanish for the $C_{\infty}$ symmetry, which we call the "chiral" components. The first three lines correspond to electric and magnetic dipolar contributions (rank-3 tensors) and the last two correspond to the electric quadrupolar terms (rank-4 tensors) which have not been considered previously.

The tensor $\chi^{e e m}$ has already been introduced by Persoons ${ }^{3}$ and utilized to interpret experimental results. ${ }^{3,5}$ Introduction of the tensor $\chi^{e e q}$ has also been proposed from a formal point of view ${ }^{11}$ but never distinguished from the previous one as far as experiments are concerned. It is one of the purpose of this paper to elucidate this point. To do so, we must not consider only the susceptibility tensors, convenient to gather the different terms contributing to the nonlinear response, but not directly accessible to a surface SHG experiment. Instead, we calculate the second harmonic electric field which is the quantity measured in the experiments. 3,5 This field originates from the far-field radiation of the lightinduced electric $(\mathbf{P})$ and magnetic $(\mathbf{M})$ polarization and from the quadrupolarization $(\mathbf{Q})$ and reads ${ }^{12}$

$$
\mathbf{E}(2 \omega) \propto\left[(\mathbf{n} \times \mathbf{P}) \times \mathbf{n}-\frac{1}{c} \mathbf{n} \times \mathbf{M}-\frac{i k}{2}(\mathbf{n} \times \mathbf{Q}(\mathbf{n})) \times \mathbf{n}\right],
$$

where $\mathbf{n}$ is a unit vector in the direction of observation and $\mathbf{Q}(\mathbf{n})$ is the tensor contraction of $\mathbf{Q}$ and $\mathbf{n}$. From the above discussion, we know that we must consider three terms in $\mathbf{P}$ $\left(\chi^{e e e}, \chi^{e e m}\right.$, and $\left.\chi^{e e q}\right)$, one in $\mathbf{M}\left(\chi^{m e e}\right)$ and one in $\mathbf{Q}$ $\left(\chi^{q e e}\right)$. With obvious notation, we therefore decompose the second harmonic electric field in

$$
\mathbf{E}(\mathbf{2} \omega)=\mathbf{E}^{e e e}+\mathbf{E}^{e e m}+\mathbf{E}^{e e q}+\mathbf{E}^{m e e}+\mathbf{E}^{q e e} .
$$

These various contributions are readily calculated from the nonlinear susceptibilities. In order to stick to the experimental situations, we also write

$$
E_{p, s}(2 \omega)=f_{p, s} E_{p}^{2}(\omega)+g_{p, s} E_{s}^{2}(\omega)+h_{p, s} E_{p}(\omega) E_{s}(\omega),
$$

where $s, p$ refer to the beam polarization. Indeed, surface SHG experiments utilizing quarter- and half-waveplates allow to measure these $f, g, h$ parameters independently. $3,5,13$ By analyzing the different terms of Eq. (2.8) in terms of Eq. (2.9), we will be able in the following to assess more pre- cisely the signature of chirality in the SHG signal and to understand better the role of quadrupolarization in it.

From the above discussion, we can define a procedure to study surface SHG from a microscopic model: (i) derive the nonlinear motion equation for the model; (ii) derive the corresponding hyperpolarizabilities; (iii) average over the isotropic layer of molecules to get the nonlinear surface susceptibilities; (iv) calculate the radiated second harmonic electric field and redistribute the different mechanisms in the $f, g$, and $h$ parameters. We will now apply this procedure to the Kuhn and Kauzmann models in the next sections.

\section{COUPLED OSCILLATOR (KUHN) MODEL}

We consider two coupled oscillators as depicted in Fig. 2. The two oscillators have the same frequency $\omega_{0}$ and the same friction coefficient $\gamma$ and are coupled through dipole-dipole interaction with a coupling parameter $\kappa$ (supposedly small). The microscopic frame is $(x, y, z)$. The oscillator $A$ is in the $x z$ plane and makes an angle $\alpha$ with the $x$-direction, its rest position is $(0,0,-d / 2)$. The oscillator $B$ is in the $y z$ plane and makes an angle $\alpha$ with the $y$-direction, its rest position is $(0,0, d / 2)$. The introduction of the angle $\alpha$ is important to get a chiral response, as it will become clear later on. This system is excited by a monochromatic wave $E e^{-i \omega t}$ which induces the oscillation of the electrons. Let us call $a e^{-i \omega t}$ (resp. $b e^{-i \omega t}$ ) the elongation of the oscillator $A$ (resp. B). The coordinates of the electron $A$

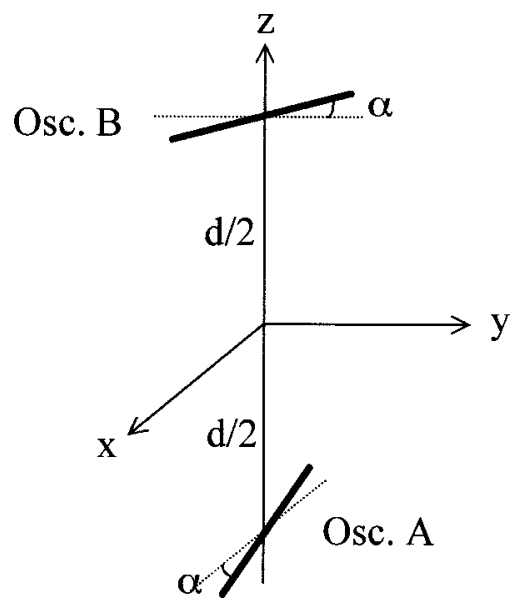

FIG. 2. Coupled oscillators used for the Kuhn model. Oscillator $A$ (resp. B) is in the $x z$ (resp. $y z$ ) plane. 
(resp. B) are $\left(a e^{-i \omega t} \cos \alpha, 0,-d / 2-a e^{-i \omega t} \sin \alpha\right) \quad[$ resp. $\left(0, b e^{-i \omega t} \cos \alpha, d / 2+b e^{-i \omega t} \sin \alpha\right)$ ]. Let us first consider the linear response of the system. The equations of motion of the electrons along their respective oscillators can be written,

$$
\begin{aligned}
D_{\omega} a^{(1)}+\kappa b^{(1)}= & \frac{-e}{m}\left[\left(E_{x}-\frac{d}{2} E_{z x}\right) \cos \alpha\right. \\
& \left.-\left(E_{z}-\frac{d}{2} E_{z z}\right) \sin \alpha\right], \\
\kappa a^{(1)}+D_{\omega} b^{(1)}= & \frac{-e}{m}\left[\left(E_{y}+\frac{d}{2} E_{z y}\right) \cos \alpha\right. \\
& \left.+\left(E_{z}+\frac{d}{2} E_{z z}\right) \sin \alpha\right],
\end{aligned}
$$

where we have introduced the following notation: $D_{\omega}=\omega_{0}^{2}$ $-\omega^{2}-2 i \omega \gamma, E_{i j}=\nabla_{i} E_{j}$, and where the superscript (1) means first-order solution. Solving this set of equations allows us to recover well-known results of this model. ${ }^{14}$ Due to the coupling, the degeneracy of the two oscillators is splitted and two new optical transitions appear with frequencies $\omega_{0}\left(1 \pm\left(\kappa / 2 \omega_{0}^{2}\right)\right)$ and opposite rotational strengths. These new states can be viewed as resulting from the in-phase and out-of-phase excitations of the two oscillators, the optical activity coming from the geometric arrangement. The introduction of the parameter $\kappa$ allows us to take empirically these effects into account. One passes from one enantiomer to the other by changing the sign of $\kappa$ and reversing one of the angles $\alpha$.

To introduce a second-order nonlinear response, we suppose that the restoring force is not harmonic and reads for oscillator $A$,

$$
-m \omega_{0}^{2} a-m \beta a^{2},
$$

the same being true for oscillator $B$. Looking for the solutions oscillating at $2 \omega$ and including the contributions coming from the development of the electric field [Eq. (2.1)], we get the following set of equations:

$$
\begin{aligned}
D_{2 \omega} a^{(2)}+\kappa b^{(2)}= & -\beta a^{(1) 2}-\frac{e}{m} a^{(1)}\left[\left(\cos \alpha E_{x x}\right.\right. \\
& \left.-\sin \alpha E_{z x}\right) \cos \alpha-\left(\cos \alpha E_{x z}\right. \\
& \left.\left.-\sin \alpha E_{z z}\right) \sin \alpha\right], \\
\kappa a^{(2)}+D_{2 \omega} b^{(2)}= & -\beta b^{(1) 2}-\frac{e}{m} b^{(1)}\left[\left(\cos \alpha E_{y y}\right.\right. \\
& \left.+\sin \alpha E_{z y}\right) \cos \alpha+\left(\cos \alpha E_{y z}\right. \\
& \left.\left.+\sin \alpha E_{z z}\right) \sin \alpha\right] .
\end{aligned}
$$

Plugging the solutions of Eq. (3.1) in Eq. (3.3), we obtain the general expressions of the second-order solutions $a^{(2)}$ and $b^{(2)}$. These solutions are used to derive the electric and magnetic dipole moments as well as the electric quadrupole one which read

$$
\begin{aligned}
& \mathbf{p}_{x}^{(2)}=-e a^{(2)} \cos \alpha, \quad \mathbf{p}_{y}^{(2)}=-e b^{(2)} \cos \alpha, \\
& \mathbf{p}_{z}^{(2)}=-e\left(b^{(2)}-a^{(2)}\right) \sin \alpha ;
\end{aligned}
$$

$$
\begin{aligned}
& \mathbf{m}_{x}^{(2)}=-\frac{i \omega d e}{2} b^{(2)} \cos \alpha, \mathbf{m}_{y}^{(2)}=-\frac{i \omega d e}{2} a^{(2)} \cos \alpha, \\
& \mathbf{m}_{z}^{(2)}=0 \\
& Q_{x x}^{(2)}=-e a^{(1) 2} \cos ^{2} \alpha, Q_{y y}^{(2)}=-e b^{(1) 2} \cos ^{2} \alpha, \\
& Q_{z z}^{(2)}=-e\left[d \sin \alpha\left(a^{(2)}+b^{(2)}\right)+\sin ^{2} \alpha\left(a^{(1) 2}+b^{(1) 2}\right)\right], \\
& Q_{x y}^{(2)}=Q_{y x}^{(2)}=0, \\
& Q_{y z}^{(2)}=Q_{z y}^{(2)}=-e\left[\frac{d}{2} b^{(2)} \cos \alpha+b^{(1) 2} \sin \alpha \cos \alpha\right], \\
& Q_{z x}^{(2)}=Q_{x z}^{(2)}=-e\left[-\frac{d}{2} a^{(2)} \cos \alpha-a^{(1) 2} \sin \alpha \cos \alpha\right] .
\end{aligned}
$$

In this last expression, we have chosen the definition $\mathbf{Q}$ $=-e \mathbf{r r}$ for which the trace is nonzero in accordance with Eq. (2.7). Utilizing the traceless form of $\mathbf{Q}$ would change the value of $\chi^{q e e}$, but would not change the radiated harmonic field, which is the relevant quantity.

Plugging the expressions of $a^{(1)}, a^{(2)}, b^{(1)}$, and $b^{(2)}$ in Eqs. (3.4)-(3.6) and utilizing Eqs. (2.3) -(2.5) allows us to calculate all the hyperpolarizabilities. General expressions are quite heavy and do not allow to extract interesting physical information. We therefore begin with some approximation and discussions. First of all, we suppose that the fundamental frequency $\omega$ is far from the oscillator frequency $\omega_{0}$. This assumption corresponds to most of the experimental cases where the SHG experiment is performed far from the electronic resonances. Note however that it does not preclude a resonance effect between an electronic transition and the harmonic frequency. This hypothesis allows us to neglect $\kappa$ compared to $D_{\omega} \approx \omega_{0}^{2}$ in our expressions. Second, examining Eq. (3.3) in detail, we see that there exists a nonlinear elongation $a^{(2)}\left[\right.$ or $\left.b^{(2)}\right]$ even if $\beta=0$, i.e., even if the oscillator is perfectly harmonic. This is due to the intrinsic nonlinearity of the electric quadrupole moment [see also Eq. (3.6)]. Looking at the general formulas (not displayed here), we observe that comparing this contribution to the anharmonic one amounts to comparing $D_{\omega}$ (or $\omega_{0}^{2}$ ) to $\beta d$. Considering the nature of electronic binding and calling $l$ the equilibrium elongation of the oscillator, one estimates that $\beta l^{2} \approx \omega_{0}^{2} l{ }^{15}$ We must therefore compare the elongation $l$ of the oscillators and the separation $d$ of the two oscillators. In order to be consistent with the hypothesis of a weak coupling and the use of dipole-dipole interaction, we suppose that $d \gg l$. This assumption is corroborated by a numerical estimation of $l$ which is in the angstrom range for electronic transitions. Making this hypothesis allows us to keep only the terms in $\beta$ in our expressions and to neglect the other ones. This assumption is very reasonable for the usual experimental configurations where the chiral molecules under study have always been chosen so as to give a high nonlinear response.

Under these two assumptions $\left(D_{\omega} \gg \kappa\right.$ and $\left.d \gg l\right)$, we get very tractable expressions for the hyperpolarizabilities. Let us now examine in detail the $\beta^{e e e}$ tensor. Out of the 27 components, there are only 6 independent ones and 3 null ones, 


$$
\begin{aligned}
\beta_{x x x}^{e e e} & =\beta_{y y y}^{e e e}=\frac{\beta e^{3} \cos ^{3} \alpha}{\epsilon_{0} m^{2} D_{\omega}^{2} D_{2 \omega}}, \\
\beta_{x x y}^{e e e} & =\beta_{x y x}^{e e e}=\beta_{y x y}^{e e e}=\beta_{y y x}^{e e e}=-\frac{\beta e^{3} \kappa \cos ^{3} \alpha}{\epsilon_{0} m^{2} D_{\omega}^{3} D_{2 \omega}}, \\
\beta_{x x z}^{e e e} & =\beta_{x z x}^{e e e}=-\beta_{y y z}^{e e e}=-\beta_{y z y}^{e e e} \\
& =\beta_{z x x}^{e e e}=-\beta_{z y y}^{e e e}=-\frac{\beta e^{3} \cos \alpha \sin ^{2} \alpha}{\epsilon_{0} m^{2} D_{\omega}^{2} D_{2 \omega}}, \\
\beta_{x z z}^{e e e} & =\beta_{z x z}^{e e e}=\beta_{z z x}^{e e e} \\
& =\beta_{y z z}^{e e e}=\beta_{z y z}^{e e e}=\beta_{z z y}^{e e e}=\frac{\beta e^{3} \cos ^{2} \alpha \sin \alpha}{\epsilon_{0} m^{2} D_{\omega}^{2} D_{2 \omega}}, \\
\beta_{x y y}^{e e e} & =\beta_{y x x}^{e e e}=-\frac{\beta e^{3} \kappa \cos ^{3} \alpha}{\epsilon_{0} m^{2} D_{\omega}^{2} D_{2 \omega}^{2}}, \\
\beta_{x y z}^{e e e} & =\beta_{x z y}^{e e e}=-\beta_{y x z}^{e e e}=-\beta_{y z x}^{e e e} \\
& =\frac{\beta e^{3} \kappa \cos ^{2} \alpha \sin \alpha}{\epsilon_{0} m^{2} D_{\omega}^{3} D_{2 \omega}^{2}}\left(D_{2 \omega}-D_{\omega}\right), \\
\beta_{z x y}^{e e e} & =\beta_{z y x}^{e e e}=\beta_{z z z}^{e e e}=0 .
\end{aligned}
$$

Examination of these coefficients is already very instructive, as one can see that some components are independent of the coupling parameter $\kappa$ whereas others are not. In fact those which depend on $\kappa$ are those for which the two space directions $x$ and $y$ come into play. This is understandable as only those components probing these two space directions will be sensitive to the coupling, and thus to the chirality of the molecules. Note that the $x y z$ components, which will prove to be relevant in the forthcoming discussion, is nonzero only under the condition that $\alpha \neq 0$ and $D_{\omega} \neq D_{2 \omega}$. Similar expressions are obtained for $\beta^{e e m}$ and $\beta^{\text {mee }}$. The number of nonzero components is higher for $\beta^{e e q}$ (41) and for $\beta^{q e e}$ (45), but the conclusions still hold; they depend on $\kappa$ if they involve $x$ and $y$.

Let us proceed now to the calculation of the nonlinear susceptibilities. Due to the high degree of symmetry of the surface $\left(C_{\infty}\right)$ and also of the individual molecules, many terms go to zero and the final results are quite simple. We give below the nonvanishing independent components for $\chi^{e e e}, \chi^{e e m}$, and $\chi^{\text {mee }}$,

$$
\begin{aligned}
\chi_{123}^{e e e}= & \frac{N e^{3} \beta}{4 \epsilon_{0} m^{2}} \cos ^{2} \alpha \sin \alpha(1+3 \cos 2 \theta) \frac{\kappa\left(D_{2 \omega}-D_{\omega}\right)}{D_{\omega}^{3} D_{2 \omega}^{2}}, \\
\chi_{132}^{e e m}= & \chi_{312}^{e e m}=\frac{i \omega N e^{3} \beta d}{8 \epsilon_{0} m^{2}} \cos ^{2} \alpha \sin \alpha(1+3 \cos 2 \theta) \\
& \times \frac{1}{D_{\omega}^{2} D_{2 \omega}}, \\
\chi_{123}^{m e e}= & -\frac{i \omega N e^{3} \beta d}{8 \epsilon_{0} m^{2}} \cos ^{2} \alpha \sin \alpha(1+3 \cos 2 \theta) \frac{1}{D_{\omega}^{2} D_{2 \omega}} .
\end{aligned}
$$

Several points are worth commenting. Due to the high degree of symmetry of the system, only the components in- volving the three space directions are nonzero. These components are null for $\alpha=0$; in that case, there cannot be any coupling between the three space directions at the molecular level and everything cancels out in the spatial averaging procedure. According to Table I, we see that the only nonvanishing electric dipolar component is chiral. This chiral component is directly proportional to $\kappa$ and vanishes for uncoupled system, as expected. Furthermore, it is proportional to $D_{2 \omega}-D_{\omega}$. This dependence comes from the $\beta_{x y z}^{e e e}$ terms, the only ones contributing to $\chi_{123}^{e e e}$ when $\varphi$ and $\psi$ are random. It means that if both the fundamental and the harmonic photon energies are far from the electronic transition, this term cancels out. Such a cancellation is expected from a general point of view: From Table I, we have $\chi_{123}^{e e e}=-\chi_{213}^{e e e}$; on the other hand, far from resonances, Kleinmann symmetry implies $\chi_{123}^{e e e}=\chi_{213}^{e e e}$, resulting in a null coefficient. It is therefore necessary to be not too far from a resonance (for example at $2 \omega$ ) to observe a SHG signal originating from chiral electric components. Let us come now to the magnetic contributions: they are achiral and independent of the coupling parameter. Note that the nonvanishing components of $\chi^{\text {eem }}$ and of $\chi^{\text {mee }}$ are equal. This is not surprising as they correspond to closely connected phenomena. All the above results are consistent with the linear response. Indeed, chirality in the Kuhn model derives not from a real magnetic moment, but rather from a $\mu \times \mu$ term $^{14}$ and is therefore a pure electric effect. This is recovered in the surface SHG where the chiral response is also a pure electric effect. Let us stress that the quadrupolar terms are not vanishing and are achiral and independent of $\kappa$.

To calculate the second harmonic electric field radiated by the molecules on the surface, we use Eq. (2.7) together with the above calculations. In order to get readable results, we perform the derivation in a simplified case. First of all, we neglect the Fresnel coefficients in the following expressions. They are only multiplicative factors that do not change the discussion. Proper introduction of these coefficients can be found in Refs. 5 and 16. Second, we assume that the incidence and reflection angles are equal to $45^{\circ}$ and that the index of refraction of the layer is equal to 1 . The geometry of the experiment is shown in Fig. 1. With these assumptions, the relevant vectors for the calculation are: $\mathbf{n}=1 / \sqrt{2}(1,0,1)$ (direction of observation), $\mathbf{k}=\omega / c \sqrt{2}(1,0,-1)$ (wave vector of the fundamental beam), $\mathbf{E}=\left(E_{p} / \sqrt{2},-E_{s}, E_{p} / \sqrt{2}\right)$ (fundamental electric field). The second harmonic electric field is expressed on the polarization basis $\hat{s}=(0,-1,0)$ and $\hat{p}$ $=1 / \sqrt{2}(-1,0,1)$. Using these definitions, we calculate the expressions of the various fields adding up to give the total second harmonic field: $\mathbf{E}(\mathbf{2} \omega)=\mathbf{E}^{e e e}+\mathbf{E}^{e e m}+\mathbf{E}^{e e q}+\mathbf{E}^{m e e}$ $+\mathbf{E}^{q e e}$ [Eq. (2.8)]. The expressions of these fields, together with their contributions to the $f, g, h$ parameters [see Eq. (2.9)] are given in Table II. These results are consistent with the conclusions drawn about the nonlinear susceptibilities. We recover that only the purely dipolar terms contribute to the chiral signal. All the magnetic dipolar or electric quadrupolar ones only give achiral signals. Furthermore, it is clear from Table II that the second harmonic fields $E^{e e m}, E^{m e e}$, $E^{e e q}$, and $E^{q e e}$ have the same order of magnitude and that they contribute to the same (achiral) coefficients $f_{p}, g_{p}$, and 
TABLE II. Second harmonic electric field radiated by an isotropic layer of molecules described by the Kuhn model. The $f_{i}$ 's are involved functions that are not given here for the sake of readability.

\begin{tabular}{|c|c|}
\hline Electric field & $\begin{array}{l}\text { Contribution } \\
\text { to } f, g, h\end{array}$ \\
\hline$E_{s}^{e e e} \propto \frac{N e^{3} \beta}{4 \epsilon_{0} m^{2}} \frac{\kappa\left(D_{2 \omega}-D_{\omega}\right)}{D_{\omega}^{3} D_{2 \omega}^{2}} \cos ^{2} \alpha \sin \alpha(1+3 \cos 2 \theta) E_{p}^{2}$ & $f_{s}$ (chiral) \\
\hline$E_{p}^{e e e} \propto \frac{N e^{3} \beta}{4 \epsilon_{0} m^{2}} \frac{\kappa\left(D_{2 \omega}-D_{\omega}\right)}{D_{\omega}^{3} D_{2 \omega}^{2}} \cos ^{2} \alpha \sin \alpha(1+3 \cos 2 \theta) E_{p} E_{s}$ & $h_{p}($ chiral $)$ \\
\hline$E_{s}^{e e m} \propto \frac{i \omega N e^{3} \beta d}{8 \epsilon_{0} m^{2} c} \frac{1}{D_{\omega}^{2} D_{2 \omega}} \cos ^{2} \alpha \sin \alpha(1+3 \cos 2 \theta) E_{p} E_{s}$ & $h_{s}$ (achiral) \\
\hline$E_{p}^{e e m} \propto \frac{i \omega N e^{3} \beta d}{8 \epsilon_{0} m^{2} c} \frac{1}{D_{\omega}^{2} D_{2 \omega}} \cos ^{2} \alpha \sin \alpha(1+3 \cos 2 \theta) E_{s}^{2}$ & $g_{p}$ (achiral) \\
\hline$E_{s}^{m e e} \propto-\frac{i \omega N e^{3} \beta d}{16 \epsilon_{0} m^{2} c} \frac{1}{D_{\omega}^{2} D_{2 \omega}} \cos ^{2} \alpha \sin \alpha(1+3 \cos 2 \theta) E_{p} E_{s}$ & $h_{s}$ (achiral) \\
\hline$E_{p}^{m e e} \propto \frac{i \omega N e^{3} \beta d}{16 \epsilon_{0} m^{2} c} \frac{1}{D_{\omega}^{2} D_{2 \omega}} \cos ^{2} \alpha \sin \alpha(1+3 \cos 2 \theta) E_{p}^{2}$ & $f_{p}$ (achiral) \\
\hline$E_{s}^{e e q} \propto-\frac{i \omega N e^{3} \beta d}{\epsilon_{0} m^{2} c} \frac{1}{D_{\omega}^{2} D_{2 \omega}} f_{1}(\theta, \alpha) E_{p} E_{s}$ & $h_{s}$ (achiral) \\
\hline$E_{p}^{e e q} \propto-\frac{i \omega N e^{3} \beta d}{\epsilon_{0} m^{2} c} \frac{1}{D_{\omega}^{2} D_{2 \omega}}\left[f_{2}(\theta, \alpha) E_{p}^{2}+f_{3}(\theta, \alpha) E_{s}^{2}\right]$ & $f_{p}, g_{p}$ (achiral) \\
\hline$E_{s}^{q e e} \propto-\frac{i \omega N e^{3} \beta d}{\epsilon_{0} m^{2} c} \frac{1}{D_{\omega}^{2} D_{2 \omega}} f_{4}(\theta, \alpha) E_{p} E_{s}$ & $h_{s}$ (achiral) \\
\hline$E_{p}^{q e e} \propto-\frac{i \omega N e^{3} \beta d}{\epsilon_{0} m^{2} c} \frac{1}{D_{\omega}^{2} D_{2 \omega}}\left[f_{5}(\theta, \alpha) E_{p}^{2}+f_{6}(\theta, \alpha) E_{s}^{2}\right]$ & $f_{p}, g_{p}$ (achiral) \\
\hline
\end{tabular}

$h_{s}$. Whereas it is not correct formally to introduce $\chi^{e e m}$, $\chi^{m e e}$ and to neglect $\chi^{e e q}, \chi^{q e e}$, it is fully justified from a practical point of view as all these terms are undistinguishable in an experiment and can be cast under generic coefficients as it is currently done. , $^{3,5}$

In this section, we have applied the general framework outlined in Sec. II to the Kuhn model of coupled oscillators. The chosen geometry (Fig. 1) is however not quite general as, for example, the two oscillators lie in perpendicular $(x z$ and $y z$ ) planes. Generalization to more complex case is possible. It is expected in such cases that the separation between chiral and achiral terms would be less strict with for example achiral contributions originating from the electric dipolar susceptibilities. The main conclusions are however not different; chiral terms from electric dipolar terms exist and magnetic dipolar and electric quadrupolar contributions are not separable from an experimental point of view. In the next section, we examine the case of an helical oscillator (Kauzmann model) which gives strikingly different results.

\section{ONE-ELECTRON (KAUZMANN) MODEL}

To study classically molecules in which the optical activity comes from a single electronic transition with electric and magnetic character, we use the model developed by Kauzmann. ${ }^{9}$ In this model, it is assumed that each molecule contains two electrons which oscillate along two identical helices having a common axis, the motion of each electron being such that they are always diametrically opposite to each other across the common axis (see Fig. 3). This model

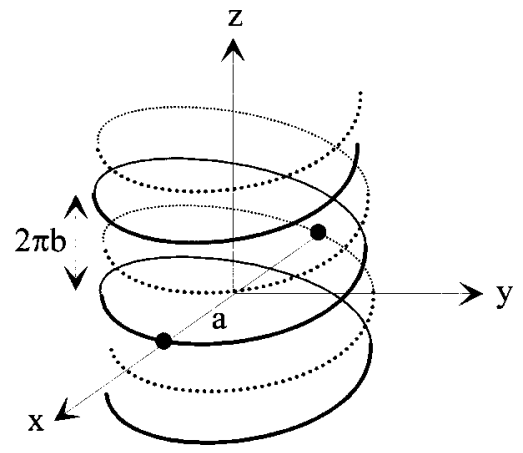

FIG. 3. Representation of the Kauzmann model. The first electron is on the solid helix and the second one on the dotted helix.

may seem artificial but it has the advantage of illustrating in a simple manner the essential feature of one-electron optical activity. By selecting the motion of the electrons along the helix axis, it raises the fundamental paradox that such helices with only one electron do not display optical activity in an isotropic arrangement ${ }^{7}$ and it makes the calculations easier and more informative. Note that a complete calculation with a more physical system (a unique electron on a helix) has been developed in Ref. 17. As discussed below, the two approaches agree on the main points.

Let us call $a$ the radius of the helix and $2 \pi b$ its pitch. We define $L=\left(a^{2}+b^{2}\right)^{1 / 2}$. The handedness of the enantiomer is determined by the parameter $b$. The coordinates of the two electrons can be described with the help of a unique parameter $\varphi$ by $(a \cos \varphi, a \sin \varphi, b \varphi)$ and $(-a \cos \varphi$, $-a \sin \varphi, b \varphi)$. We suppose that these two electrons are bound through an oscillator at frequency $\omega_{0}$ with a friction constant $\gamma$. Exciting the first electron with a monochromatic wave $E e^{-i \omega t}$, we get the following first-order equation of motion: ${ }^{17}$

$$
D_{\omega} \varphi=\frac{-e}{m L} E_{t}
$$

where $D_{\omega}=\omega_{0}^{\prime 2}-\omega^{2}-2 i \omega \gamma\left(\omega_{0}^{\prime}=\omega_{0} b / L\right) . E_{t}$ is the tangential component of the electric field acting on the first electron. It can be expressed as a function of the coordinates of the field as

$$
E_{t}=-\frac{a}{L} \sin \varphi E_{x}+\frac{a}{L} \cos \varphi E_{y}+\frac{b}{L} E_{z} .
$$

Nonlocal response is considered by expanding the electric field around the center of the frame according to Eq. (2.1). When taking into account the equation of the second electron, several terms cancel out and Eq. (4.1) reduces to

$$
D_{\omega} \varphi^{(1)}=\frac{-e}{m L}\left(\frac{b}{L} E_{z}+\frac{a^{2}}{L^{2}} E_{x y}\right),
$$

where here again, the superscript (1) means first-order solution. To access the second-order nonlinear response, we suppose that the oscillators are anharmonic [Eq. (3.2)] and we look for the solution oscillating at $2 \omega$. The corresponding equation is 


$$
\begin{aligned}
D_{2 \omega} \varphi^{(2)}= & -\beta \varphi^{(1) 2}-\frac{e a^{2}}{m L^{2}} \varphi^{(1)}\left(-E_{x x}+E_{y y}\right) \\
& -\frac{e b^{2}}{m L^{2}} \varphi^{(1)} E_{z z} .
\end{aligned}
$$

From these equations, it is straightforward to obtain the second-order induced electric and magnetic dipole moments as well as the electric quadrupole one which read here,

$$
\begin{aligned}
& \mathbf{p}^{(2)}=-2 e b \varphi^{(2)} \hat{\mathbf{z}}, \\
& \mathbf{m}^{(2)}=2 i \omega e a^{2} \varphi^{(2)} \hat{\mathbf{z}}, \\
& \mathbf{Q}^{(2)}=-e\left(\begin{array}{ccc}
2 a^{2} \varphi^{(1) 2} & 2 a^{2} \varphi^{(2)} & 0 \\
2 a^{2} \varphi^{(2)} & 2 a^{2} \varphi^{(1) 2} & 0 \\
0 & 0 & 2 b^{2} \varphi^{(1) 2}
\end{array}\right),
\end{aligned}
$$

and from which we deduce the expressions of the hyperpolarizabilities. The number of nonzero elements is reduced in this model thanks to Kauzmann's simplifying assumptions and we get

$$
\begin{aligned}
& \beta_{z z z}^{e e e}=\frac{2 \beta e^{3} b^{3}}{\epsilon_{0} m^{2} L^{4} D_{\omega}^{2} D_{2 \omega}}, \\
& \beta_{z z z}^{e e m}=-\frac{2 i \omega \beta e^{3} a^{2} b^{2}}{\epsilon_{0} m^{2} L^{4} D_{\omega}^{2} D_{2 \omega}}, \\
& \beta_{z z z}^{\text {mee }}=-\frac{2 i \omega \beta e^{3} a^{2} b^{2}}{\epsilon_{0} m^{2} L^{4} D_{\omega}^{2} D_{2 \omega}},
\end{aligned}
$$

and $\beta_{z z x x}^{e e q}=-\beta_{z z y y}^{e e q}, \beta_{z z x y}^{e e q}=\beta_{z z y x}^{e e q}, \beta_{z z z z}^{e e q}$ as nonvanishing components, all the $\beta^{\text {qee }}$ being null. As for Kuhn's model, some of the quadrupole components do not depend on the nonlinear parameter $\beta$ but we do not need to neglect them at this point as they will prove not to contribute in the following.

Expressions of the nonlinear susceptibilities obtained after averaging over a $C_{\infty}$ distribution are readily obtained. Here are the nonzero independent components,

$$
\begin{aligned}
& \chi_{113}^{e e e}=\chi_{311}^{e e e}=\frac{N \beta e^{3} b^{3} \cos \theta \sin ^{2} \theta}{\epsilon_{0} m^{2} L^{4} D_{\omega}^{2} D_{2 \omega}}, \\
& \chi_{333}^{e e e}=\frac{2 N \beta e^{3} b^{3} \cos ^{3} \theta}{\epsilon_{0} m^{2} L^{4} D_{\omega}^{2} D_{2 \omega}} ; \\
& \chi_{113}^{e e m}=\chi_{311}^{e e m}=\frac{-i \omega N \beta e^{3} a^{2} b^{2} \cos \theta \sin ^{2} \theta}{\epsilon_{0} m^{2} L^{4} D_{\omega}^{2} D_{2 \omega}}, \\
& \chi_{333}^{e e m}=\frac{-2 i \omega N \beta e^{3} a^{2} b^{2} \cos ^{3} \theta}{\epsilon_{0} m^{2} L^{4} D_{\omega}^{2} D_{2 \omega}} ; \\
& \chi_{113}^{m e e}=\chi_{311}^{m e e}=\frac{-i \omega N \beta e^{3} a^{2} b^{2} \cos \theta \sin ^{2} \theta}{\epsilon_{0} m^{2} L^{4} D_{\omega}^{2} D_{2 \omega}}, \\
& \chi_{333}^{m e e}=\frac{-2 i \omega N \beta e^{3} a^{2} b^{2} \cos ^{3} \theta}{\epsilon_{0} m^{2} L^{4} D_{\omega}^{2} D_{2 \omega}} .
\end{aligned}
$$

Examination of Table I shows that $\chi^{e e e}$ has only achiral components, whereas $\chi^{e e m}$ and $\chi^{\text {mee }}$ has only chiral ones. This is in good agreement with the linear response; chirality
TABLE III. Second harmonic electric field radiated by an isotropic layer of molecules described by the Kauzmann model.

\begin{tabular}{ll}
\hline \hline \multicolumn{1}{c}{ Electric field } & $\begin{array}{c}\text { Contribution } \\
\text { to } f, g, h\end{array}$ \\
\hline$E_{s}^{e e e} \propto-\frac{\sqrt{2} N e^{3} \beta b^{3}}{\epsilon_{0} m^{2} L^{4}} \frac{1}{D_{\omega}^{2} D_{2 \omega}} \cos \theta \sin ^{2} \theta E_{p} E_{s}$ & $h_{s}$ (achiral) \\
$E_{p}^{e e e} \propto \frac{\sqrt{2} N e^{3} \beta b^{3}}{8 \epsilon_{0} m^{2} L^{4}} \frac{1}{D_{\omega}^{2} D_{2 \omega}} \cos \theta\left[(1+3 \cos 2 \theta) E_{p}^{2}\right.$ & $f_{p}, g_{p}$ (achiral) \\
$\left.+4 \sin ^{2} \theta E_{s}^{2}\right]$ & \\
$E_{s}^{e e m} \propto \frac{\sqrt{2} i \omega N e^{3} \beta a^{2} b^{2}}{\epsilon_{0} m^{2} L^{4} c} \frac{1}{D_{\omega}^{2} D_{2 \omega}} \cos \theta \sin ^{2} \theta\left[E_{p}^{2}-E_{s}^{2}\right]$ & $f_{s}, g_{s}$ (chiral) \\
$E_{p}^{e e m} \propto \frac{\sqrt{2} i \omega N e^{3} \beta a^{2} b^{2}}{8 \epsilon_{0} m^{2} L^{4} c} \frac{1}{D_{\omega}^{2} D_{2 \omega}}(3 \cos \theta+5 \cos 3 \theta) E_{p} E_{s}$ & $h_{p}$ (chiral) \\
$E_{s}^{m e e} \propto \frac{\sqrt{2} i \omega N e^{3} \beta a^{2} b^{2}}{8 \epsilon_{0} m^{2} L^{4} c} \frac{1}{D_{\omega}^{2} D_{2 \omega}} \cos \theta\left[(1+3 \cos 2 \theta) E_{p}^{2}\right.$ & \\
$\left.+4 \sin ^{2} \theta E_{s}^{2}\right]$ & $f_{s}, g_{s}$ (chiral) \\
$E_{p}^{m e e} \propto-\frac{\sqrt{2} i \omega N e^{3} \beta a^{2} b^{2}}{\epsilon_{0} m^{2} L^{4} c} \frac{1}{D_{\omega}^{2} D_{2 \omega}} \cos \theta \sin ^{2} \theta E_{p} E_{s}$ & \\
\hline \hline
\end{tabular}

is clearly due to the magnetic moment induced by the helical motion of the electrons. An interesting point is that the quadrupolar terms $\chi^{e e q}$ and $\chi^{q e e}$ are identically null. Reducing the nonlocal effects to the magnetic ones is therefore fully correct for this model. Considering now the radiated second harmonic electric field, we recover the expressions previously obtained for magnetic terms. ${ }^{3,5}$ They are given in Table III for the same geometry as Fig. 1. $E^{e e e}$ contribute only to $f_{p}, g_{p}$, and $h_{s}$ (achiral), whereas $E^{e e m}$ and $E^{\text {mee }}$ contribute only to $f_{s}, g_{s}$, and $h_{p}$ (chiral). Note that the exact cancellation of the chiral electric contribution is a consequence of the Kauzmann model which removes the contributions of the electric dipole in the surface plane. However, considering the calculation in Ref. 17 with a unique electron on a helix, one can show that the chiral electric susceptibility components give a negligible effect as soon as the optical nonlinear response is strong enough. Working in the same framework as for the Kuhn model, we therefore obtain a quite good agreement with Ref. 17.

Kauzmann model allows to obtain simple results and to show that the chiral signal in surface SHG originates in the magnetic terms and that electric quadrupolar susceptibilities vanish. This in in complete contrast with the Kuhn model introduced in the previous section. These two models are therefore very complementary. We will now examine previously obtained experimental results in the light of these two models.

\section{DISCUSSION}

Before discussing the experimental results, we would like to stress again the difference between the two models. In the Kuhn model, optical activity occurs because the two electric dipole moments are arranged in a nonmirrorsymmetrical configuration. This is known to be the main mechanism in molecules constituted of two (or more) mono- 
mers in interaction. ${ }^{18,19}$ As a consequence, we find that optical activity in surface SHG is also due to purely electric terms. On the other hand, optical activity in the Kauzmann model is due to the helical path imposed to the electrons by the molecular backbone. It is clear here that excitation of an electric dipole moment will induce a magnetic moment and vice versa ${ }^{9}$ and that optical activity is mainly a magnetic effect. This is true also for second-order nonlinear optics. The two models depicted above, although simple, give a very physical approach of the microscopic origins of optical activity and of their consequences for surface SHG.

Let us consider now the interchange of the handedness of the enantiomers. Interchanging the two enantiomers by mirror imaging of the system leads to changing the sign of the chiral parameter and of the nonlinear coupling term together. This last feature can be understood as follows: looking at Fig. 3, one sees that a positive $\beta$ means that the oscillator is stiffer in the $y>0$ direction compared to the $y<0$ one, i.e., when the electrons go upward. Taking the mirror image of the helix, the oscillator must still be stiffer for electrons going upward, i.e., for $y<0$. This is possible only if $\beta$ changes sign when going from an enantiomer to the other one. Applying these changes shows that the $\chi^{e e e}$ tensor does not change sign whereas $\chi^{e e m}$ and $\chi^{\text {mee }}$ do for the Kauzmann model. Applying similar rules to the Kuhn model (which is somewhat more involved as one must change concommitantly the sign of the angle $\alpha$ and of the nonlinear parameter $\beta$ for one of the two oscillators), one gets the opposite result. However, the only nonvanishing components of $\chi^{e e m}$ and $\chi^{\text {mee }}$ (resp. $\chi^{e e e}$ ) are chiral (resp. achiral) in the Kauzmann model while they are achiral (resp. chiral) in the Kuhn one (cf. Tables II and III). Finally, both models are consistent with the very general symmetry rules of chirality in two dimensions, ${ }^{20}$ chiral components change sign, whereas achiral ones do not when interchanging enantiomers. As the components which change sign with the enantiomers are the electric ones in the Kuhn model and the magnetic ones in the Kauzmann model, this should be observed in an experiment measuring the absolute phase of the susceptibility tensors. However, experiments have only given relative phases (if any) so far and could not access this information.

We can also estimate from our calculations the relative magnitudes of the radiated second harmonic fields so as to compare the chiral and achiral contributions. Such a comparison is straightforward for the Kauzmann case. Looking at Table III, one gets that the chiral contributions over the achiral ones scale as $a^{2} / \lambda b$. The molecular parameters $a$ et $b$ being in the same range, but in any case, much smaller than the wavelength, this ratio is clearly much smaller than 1 . As an example, we consider hexahelicene for which $a=2.50 \AA$ and $b=0.605 \AA{ }^{21}$ and we get a ratio of $10^{-3}$ for a wavelength of $1 \mu \mathrm{m}$. This is quite consistent with the fact that the chiral parts come from magnetic effects whereas the achiral ones come from electric effect, and one recovers the usual ratio between both contributions. In the Kuhn model, such a comparison is not so simple. Indeed, two contradictory features occur. On one hand, the chiral part being of electric origin, it is expected to be larger that the achiral part, of magnetic origin. On the other hand, the chiral part is non zero only if at least one of the frequencies is close to a molecular resonance. The ratio between chiral and achiral parts will therefore not depend only on geometrical factors of the molecule, as for the Kauzmann model, but will also be very sensitive to the excitation photon energies compared to the electronic or vibrational transitions of the molecule. In particular, if the second harmonic frequency is close to a resonance, the ratio chiral/achiral scales as $\left(\kappa / D_{2 \omega}\right)$ $\times(\lambda / d)$ and can become quite large. As discussed below, this has been experimentally demonstrated in helicenebisquinone where the chiral electric contributions dominate. ${ }^{22}$

We can now proceed to an examination of previously published experimental results in view of our calculations. Considering the very simple models we have introduced, it is not possible to look for more than a qualitative agreement. However, we will see that our calculations allow to reconcile controversial interpretations that were given in the past about the origin of the chiral response in surface SHG experiments. In a pionneering work, Byers et al. ${ }^{2}$ demonstrated SHG from a surface of chiral 2,2'-dihydroxy-1,1'binaphtyl. They clearly evidenced chiral effects in the harmonic signal (namely, a rotation of the polarization of the harmonic beam compared to the fundamental one) which they explain by considering only the electric dipolar nonlinear susceptibility. More recently, Van Elshocht et al. ${ }^{23}$ confirmed this result in a helical polymer based on binaphtyl monomers where no indication of magnetic contributions were observed. Similar results were obtained in Langmuir-Blodgett films of a chiral helicenebisquinone derivative which give very strong SHG signal. ${ }^{22}$ Here again, no evidence of magnetic contributions was found and the dominant contribution was $\chi_{123}^{e e e}$. This chiral contribution was measured to be at least 6 times larger than the achiral ones. These three experiments rely on similar molecules. In every case, the molecules under consideration are composed of two identical monomers spatially arranged in a nonmirror symmetric configuration. Optical activity in such molecules clearly originates from excitonic coupling ${ }^{18}$ and therefore should be correctly described by coupled oscillators. The experimental results are consistent with the above calculation for the Kuhn model in which the electric terms are dominant. In particular, the helicenebisquinone compounds seem to be very well fitted to this model which implies that if the second harmonic frequency is not too far from an electronic resonance, the magnetic terms are negligible compared to the electric ones. This looks quite appropriate to the helicenebisquinone molecule.

On the other hand, several experiments have proven that in some cases, the electric-dipole approximation is not sufficient for explaining SHG on a surface of chiral molecules. ${ }^{4,5}$ More strikingly, there exist molecules for which the chiral components are dominated by the magnetic ones. It is the case of polythiophene ${ }^{4}$ or of chiral poly(isocyanide) films for which the largest chiral magnetic component, of the order of $20 \%$ of the largest achiral electric one, is three times as big as the largest chiral electric one. ${ }^{24}$ In such molecules, the nonlinearity originates in the delocalization of $\pi$ electrons along a linear path which is slightly twisted due to the presence of a chiral center. This motion is comparable to the motion of an electron on a helix and can therefore be mod- 
elized by the Kauzmann model. Predominance of the magnetic terms in the chiral response is expected from the above calculations.

In the general case, molecules cannot be modelized by a unique model. This is the case for example in Ref. 5 where the molecular backbone is not strictly linear and where many susceptibility components are expected to play a role. In that case, all the effects are mixed up and chiral components were measured to have similar orders of magnitude for the electric or magnetic contributions. However, by choosing the molecular structure, one can favor the electric or the magnetic origin in the chiral SHG signal.

Before concluding, we would like to further comment on the helicenebisquinone case. ${ }^{22}$ The experiments were performed on Langmuir-Blodgett films and it was shown that enantiomerically-pure molecules were stacked in helical supramolecular aggregates. There seems to be a competition between the molecular level which pertains to the Kuhn model and the supramolecular one which introduces a helical path. It is experimentally clear that the Kuhn model is well fitted, which means that the chiral nonlinear response is dominated by the molecular level. The supramolecular organization, although not prevalent as far as chirality is concerned, is nevertheless important as it allows the various molecular entities to radiate in a coherent way and to interfere constructively.

\section{CONCLUSION}

In this paper, we have made use of two classical models of the origin of the optical activity in chiral molecules and extended them to the second-order nonlinear response in order to understand better surface SHG experiments. The first model is the Kuhn one in which the optical activity comes from the coupling of two oscillators. The second one, introduced by Kauzmann, describes an electron harmonically bound on a helix. By allowing the oscillators to be anharmonic, we are able to calculate the second-order hyperpolarizabilities of the molecules, including the nonlocal (magnetic dipolar and electric quadrupolar) response. From these coefficients, we get the nonlinear susceptibilities of an assembly of molecules deposited on a surface and derive the radiated second harmonic field, as measured in the experiments. The two models give strikingly different results. In the Kuhn model, the chiral response comes from the electronic susceptibility, whereas in the Kauzmann case, it comes from the magnetic ones. Furthermore, we show that the electric quadrupolar contributions are not negligible in the Kuhn model, but that they are indiscernible from the magnetic dipolar ones and that it is therefore legitimate to cast both effects under a generalized magnetic term.
This calculation allows to understand better previously published experimental results on SHG on a layer of chiral molecules where various interpretations relying either on purely electric effects or on magnetic ones were given. Indeed, depending on the molecular structure, one of the two models is more appropriate and our calculation predicts that the chiral signatures in the SHG signal are very different. A close analysis of previous experiments shows that their results are consistent with our modelizations.

This calculation, although simple, brings very instructive information about the link between the molecular structure and the surface SHG signal. A contrario, it shows the power of surface SHG to study the origin of chirality in molecules. By measuring separately the local/nonlocal susceptibility components as well as the achiral/chiral ones and by comparing with the results of the model calculations, it allows us to get a more precise understanding of the microscopic mechanisms of chirality.

${ }^{1}$ T. Petralli-Mallow, T. M. Wong, J. D. Byers, H. I. Yee, and J. M. Hicks, J. Phys. Chem. 97, 1383 (1993).

${ }^{2}$ J. D. Byers, H. I. Yee, and J. M. Hicks, J. Chem. Phys. 101, 6233 (1994).

${ }^{3}$ M. Kauranen, T. Verbiest, J. J. Maki, and A. Persoons, J. Chem. Phys. 101, 8193 (1994).

${ }^{4}$ S. Van Elshocht, T. Verbiest, M. Kauranen, B. M. W. Langeveld-Voss, and E. W. Meijer, J. Chem. Phys. 107, 8201 (1997).

${ }^{5}$ M. C. Schanne-Klein, F. Hache, A. Roy, C. Flytzanis, and C. Payrastre, J. Chem. Phys. 108, 9436 (1998).

${ }^{6}$ M. Kauranen, T. Verbiest, and A. Persoons, J. Nonlinear Opt. Phys. Mater. 8, 171 (1999).

${ }^{7}$ E. U. Condon, Rev. Mod. Phys. 9, 432 (1937).

${ }^{8}$ W. Kuhn, Z. Phys. Chem. Abt. B 4, 14 (1929).

${ }^{9}$ W. Kauzmann, Quantum Chemistry (Academic, New York, 1957), p. 616.

${ }^{10}$ Y. R. Shen, Annu. Rev. Phys. Chem. 40, 321 (1989).

${ }^{11}$ M. Kauranen, T. Verbiest, and A. Persoons, J. Mod. Opt. 45, 403 (1998).

${ }^{12}$ J. D. Jackson, Classical Electrodynamics, 3rd ed. (Wiley, New York, 1999), p. 407.

${ }^{13}$ F. Geiger, R. Stolle, G. Marowsky, M. Palenberg, and B. U. Felderhof, Appl. Phys. B: Lasers Opt. 61, 135 (1995).

${ }^{14}$ S. F. Mason, Molecular Optical Activity and the Chiral Discrimination (Cambridge University Press, Cambridge, 1982), p. 42.

${ }^{15}$ N. Bloembergen, Nonlinear Optics (Benjamin, New York, 1965), p. 7.

${ }^{16}$ J. J. Maki, M. Kauranen, and A. Persoons, Phys. Rev. B 51, 1425 (1995).

${ }^{17}$ J. J. Maki and A. Persoons, J. Chem. Phys. 104, 9340 (1996).

${ }^{18}$ E. L. Eliel and S. H. Wilen, Stereochemistry of Organic Compounds (Wiley, New York, 1994), p. 1043.

${ }^{19}$ C. R. Cantor and P. R. Schimmel, Biophysical Chemistry (Freeman, San Francisco, 1980), Part II, p. 421.

${ }^{20}$ L. Hecht and L. D. Barron, Mol. Phys. 89, 61 (1996).

${ }^{21}$ I. Tinoco and R. W. Woody, J. Chem. Phys. 40, 160 (1964).

${ }^{22}$ C. Nuckolls, T. J. Katz, S. Van Elshocht, T. Verbiest, H. G. Kuball, S. Kiesewalter, A. J. Lovinger, and A. Persoons, J. Am. Chem. Soc. 121, 3453 (1999).

${ }^{23}$ S. Van Elshocht, T. Verbiest, M. Kauranen, L. Ma, H. Cheng, K. Y. Musick, L. Pu, and A. Persoons, Chem. Phys. Lett. 309, 315 (1999).

${ }^{24}$ M. Kauranen, J. J. Maki, T. Verbiest, S. Van Elshocht, and A. Persoons, Phys. Rev. B 55, 1985 (1997). 